\title{
Análise espaço-temporal da incidência de tuberculose na atenção primária
}

\author{
Space-temporal analysis of the incidence of tuberculosis in primary care \\ Bruno de Oliveira Santos', Thuany Vulcão Raniéri Brito ${ }^{2}$, Cristal Ribeiro Mesquita ${ }^{3}$, \\ Ricardo José de Paula Souza e Guimarães ${ }^{4}$, Lucas de Araújo Leão², Mateus Pantoja Rocha²
}

Resumo Objetivo: Analisar a distribuição espacial das incidências de Tuberculose (TB) nas áreas adscritas de uma Unidade de Saúde da Família, no município de Belém-PA, no período de 2010 a 2015. Método: Estudo ecológico descritivo retrospectivo com abordagem quantitativa. A coleta de dados foi realizada junto à Secretária Municipal de Saúde de Belém. Foi utilizada técnica estatística, qui-quadrado de partição para evidenciar a significância entre possíveis proporções desiguais das variáveis e a geoestatistica, por meio da técnica de Kernel que permitiu detectar onde os eventos estavam concentrados. Resultados: A análise dos dados revelou a maior ocorrência de casos no ano de 2012 (25,1\%) e a menor em 2010 (6,8\%). Observou-se a maior prevalência de TB no sexo masculino (70,45\%), cor parda (77,3\%), faixa etária entre $14-25$ anos (27,3\%) e escolaridade até o ensino fundamental incompleto (22,7\%). Apesar da contribuição dos Agentes Comunitários de Saúde (ACS) na localização dos endereços, apenas foi possível geocodificar $95 \%$ dos casos novos. Foi observado também que a doença está distribuida de forma heterogênea, evidenciando desigualdades nas áreas analisadas, com aglomeração espacial dos casos em áreas tradicionalmente mais carentes da região. O estudo proporcionou o conhecimento Epidemiológico da área, fornecendo elementos para construção da cadeia explicativa dos problemas encontrados, auxiliando na tomada de decisão dos gestores públicos. Conclusão: As técnicas utilizadas permitiram identificar áreas de vulnerabilidade ao adoecimento, e seu reconhecimento é imprescindível para direcionar políticas públicas de enfrentamento e controle da doença.

Descritores: saúde pública; tuberculose; mapeamento geográfico.

Summary Purpose: To analyze the spatial distribution of the incidence of tuberculosis (TB) in the adscritas areas of a family health unit, in the municipality of Belém-PA, in the period of 2010 to 2015. Methods: Retrospective ecological descriptive study with quantitative approach. The data collection was held next to the Municipal Health Secretary of Bethlehem. Statistical technique was used, chi-squared partition to highlight the significance between possible unequal dimensions of variables and geostatistical, through the Kernel technique that allowed to detect where the events were concentrated. Results: Data analysis revealed the largest occurrence of cases in the year of 2012 (25.1\%) and the smallest in 2010 (6.8\%). The highest prevalence of male TB (70.45\%) was observed, Brown (77.3\%), age between 14-25 years (27.3\%) and schooling until incomplete elementary school (22.7\%). Despite the contribution of the Community Health Agents (ACS) to the location of the addresses, it was only possible to GeoCode $95 \%$ of the new cases. It has also been observed that the disease is distributed in a heterogeneous way, demonstrating inequalities in the analyzed areas, with spatial agglomeration of cases in traditionally more deprived areas of the region. The study provided the epidemiological knowledge of the area, providing elements to construct the explanatory chain of the problems encountered, aiding in the decision-making of public managers. Conclusion: The techniques used have allowed to identify areas of vulnerability to the illness, and their recognition is indispensable to direct public policies to confront and control the disease.

Keywords: public health; tuberculosis; geographic mapping.

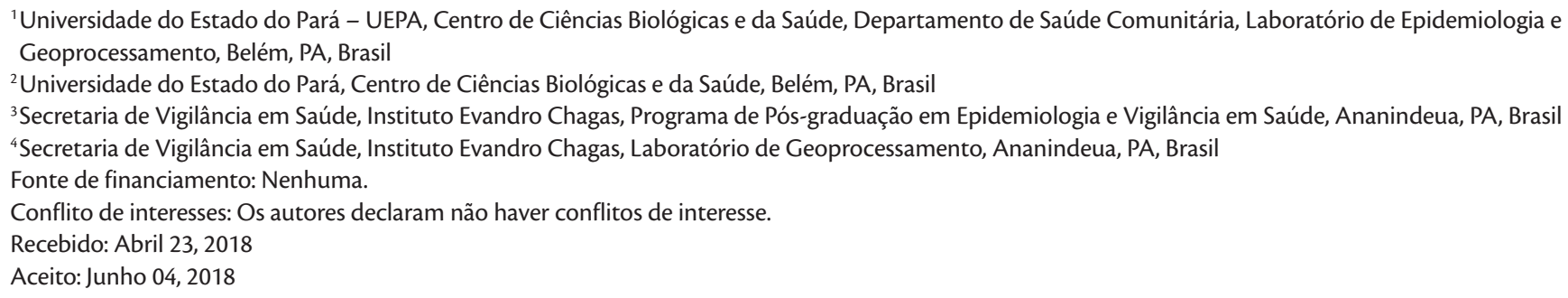

Trabalho realizado na Universidade do Estado do Pará - UEPA, Belém, PA, Brasil. 


\section{Introdução}

A Tuberculose (TB), apesar de ser conhecida com uma doença antiga, ainda é considerada um problema de saúde pública mundial, causando em torno de 5 mil mortes por dia globalmente ${ }^{1}$, estando associada aos indicadores sociais ${ }^{2}$. A existência de serviços de saúde inadequados, de regiões de pobreza, e as condições de vida contribuem para que áreas se tornem vulneráveis ao aparecimento de doenças transmissíveis, como a $\mathrm{TB}^{3}$.

Segundo a Organização Mundial de Saúde (OMS), em 2014 foram notificados 9 milhões de novos casos de TB no mundo ${ }^{4}, 71.230$ casos novos no Brasil, sendo 3.464 no estado do Pará, ocupando o terceiro lugar em taxa de incidência de TB na região norte, com o valor de 39,2/100 mil habitantes ${ }^{5}$. Nesse mesmo ano, o município de Belém, apresentou taxa de incidência de 83,2/100 mil habitantes, sendo responsável pela metade dos novos casos no Pará.

Acredita-se que na maioria dos casos, a TB pode ter resolutividade na Atenção Primária em Saúde (APS), e equipes de saúde capacitadas podem interferir positivamente para melhor controle da doença, por meio da busca ativa dos sintomáticos respiratórios ${ }^{6,7}$. Nessa perspectiva, percebe-se a importância de serviços de acesso à saúde próximos às populações de um determinado território, com ações resolutivas, de controle e de informações ${ }^{7}$.

A análise espacial em saúde é uma ferramenta importante para a tomada de decisão e para o planejamento, possibilitando aos gestores da área da saúde desenhar políticas e programas para o controle da $\mathrm{TB}^{8}$. Nesse processo, o uso do mapeamento geográfico, que é uma técnica da análise espacial capaz de coletar, tratar e manipular dados espaciais, é de grande relevância na avaliação de risco em saúde, ao possibilitar o mapeamento de doenças ${ }^{9,10}$.

Destarte, a ocorrência de alta incidência de casos de TB, nas últimas décadas, representa um grande desafio para a saúde pública. Dessa forma, a possibilidade do mapeamento geográfico poderia auxiliar as políticas de controle da doença ${ }^{11,12}$. Assim, o presente estudo objetivou analisar a distribuição espacial das incidências de TB nas áreas adscritas à USF Eduardo Angelim, no município de Belém-PA, no período de 2010 a 2015.

\section{Métodos}

Foi realizado um estudo ecológico, descritivo, retrospectivo com abordagem quantitativa, realizado por meio do mapeamento geográfico da incidência de TB, conforme casos novos notificados no período de 2010 a 2015, na USF Eduardo Angelim, que se localiza no bairro Parque Guajará, no município de Belém-Pará. A amostra do estudo foi de 44 casos de TB.

A coleta de dados foi realizada utilizando fonte secundária, obtidos junto à Secretaria Municipal de Saúde (SESMA). Os dados foram organizados em uma planilha no Microsoft ${ }^{\circ}$ Office Excel ${ }^{\circ} 2010$, conforme as variáveis de sexo, idade, raça/cor e escolaridade.

O geoprocessamento e análise dos dados foram realizados em quatro etapas:

A primeira etapa levou em consideração a aquisição dos dados de notificação, fornecida pelo Sistema de Informação de Agravos de Notificação (SINAN) do DATASUS; bases cartográficas da Companhia de Desenvolvimento e Administração da Área Metropolitana de Belém (CODEM) e do Instituto Brasileiro de Geografia e Estatística (IBGE); populacionais do IBGE; e imagens do Google Earth de julho de 2015.

Na segunda etapa foi realizada a depuração dos dados, possibilitando retirar possíveis fatores causadores de vieses a partir da utilização do programa Microsoft Excel 2010. Este processo objetivou a implementação de um modelo de um Banco de Dados Geográfico (BDGeo) dos casos notificados. Na terceira etapa, foi realizado o georreferenciamento, obtenção das coordenadas (latitude e longitude), utilizando o software Google Earth. Posteriormente, foram criadas as bases cartográficas das três áreas de assistência das Equipes de Saúde da Família, a saber, Área 1: Eduardo Angelim, Área 2: Café Liberal e Área 3: Fé em Deus.

O BDGeo e as bases de dados dos limites de atuação de cada USF, ambas em formato vetorial, foram importadas para um Sistemas de Informação Geográfica (SIG), que possibilitou a integração das mesmas utilizando a ferramenta join do software ArcGis 10.2 e a expressão visual em termos de mapas digitais no software TerraView e ArcGis. 
Para encontrar o coeficiente de deteç̧ão dos casos de Tuberculose foi usado o parâmetro de coeficiente de incidência utilizado pela Organização Mundial de Saúde-OMS/DATASUS, da qual se divide o número de casos de TB pelo número da população as áreas da USF e multiplica-se por 10.000.

A quarta e última etapa foi relacionada à análise dos valores do Coeficiente de Incidência obtidos, por área de USF, assim sendo, foi utilizada a técnica estatística qui-quadrado de partição, para evidenciar a significância entre possíveis proporções desiguais das variáveis inicialmente classificados em cinco categorias: Baixo (menor que 2,0); Médio (2,0 a 3,9); Alto (4,0 a 6,9); Muito Alto (7,0 a 9,9) e Hiperendêmico (igual ou maior que 10,0$)$.

Na sequência foi utilizado o interpolador de densidade Kernel do software TerraView com raio adaptativo e função quártica. O kernel é uma técnica da geoestatística utilizada para analisar o comportamento de padrões de pontos, fornecendo, por meio de interpolação, a intensidade pontual do processo em toda a região estudada, o qual permitiu detectar onde os eventos estavam concentrados.

O estudo cumpriu com as exigências éticas, obedecendo à Declaração de Helsinque, ao Código de Nuremberg e às normas da Resolução ${ }^{\circ}$ 466/12 do Conselho Nacional de Saúde. A pesquisa obteve parecer favorável do Comitê de Ética em Pesquisa no dia 08 de novembro de 2016 sob CAAE n ${ }^{\circ}$ 59074116.0.0000.5174.

\section{Resultados}

Dentre os 44 casos notificados no período de 2010 a 2015, o maior número de casos foi verificado no ano de 2012 (25,1\%) e o menor em 2010 (6,8\%). Sendo a área Café Liberal com maior número de casos, sendo responsável por $40,9 \%$ das notificações.

Por meio dos cálculos das taxas de incidência de TB observou-se que houve variações na incidência no período de 2010 a 2015. A menor e a maior taxa foram verificadas na área Eduardo Angelim no ano de 2011 e 2013, respectivamente ( $0 \%$ e $11,8 \%)$.

A Figura 1 mostra a área de estudo com a distribuição espacial dos 44 casos de TB na USF Eduardo Angelim e as áreas de assistência das Equipes de Saúde da Família.

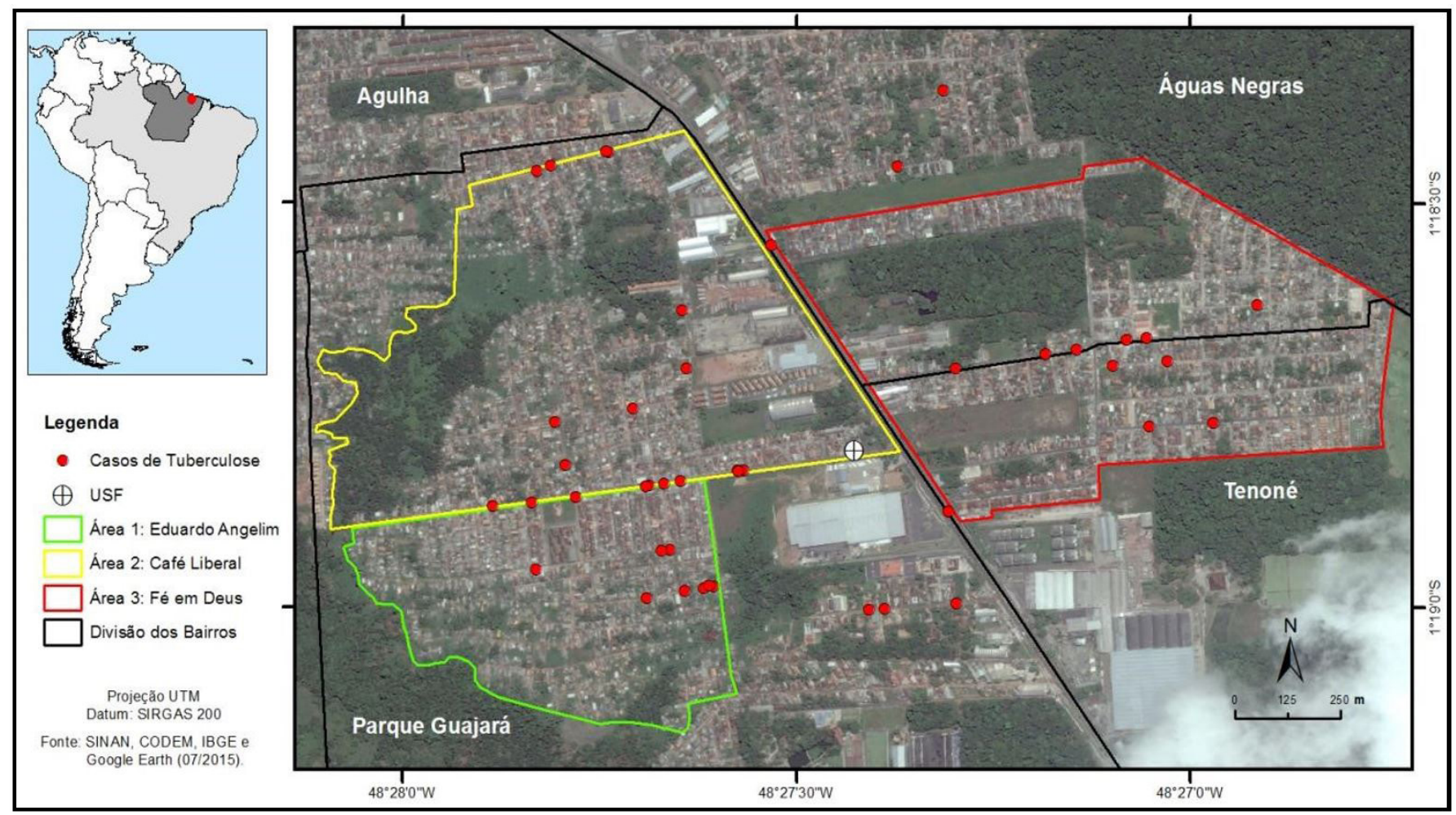

Figura 1. Distribuição espacial dos casos de TB notificados e residentes nas áreas adscritas à USF Eduardo Angelim, no município de Belém-PA, no período de 2010 a 2015.

Fonte: EPIGEO/DSCM/CCBS/UEPA, 2016. 
A caracterização da população estudada no período de seis anos demonstra que a TB foi mais prevalente no sexo masculino (31 casos /70,45\%), cor parda (34 casos /77,3\%), faixa etária entre 14-25 anos (12 casos /27,3\%) e escolaridade até o ensino fundamental incompleto (10 casos/22,7\%) (Tabela 1).

A Figura 2 representa a aplicação da ferramenta Densidade Kernel, demonstrando as áreas com maiores riscos de transmissão de TB, representadas por cores mais quentes. Assim, verifica-se maior risco nas áreas 1 (Eduardo Angelim) e 2 (Café Liberal).

\section{Discussão}

A população em estudo apresenta predominância do sexo masculino, adulto jovem, com baixa escolaridade. A diferença de exposição a fatores de risco entre homens e mulheres é um aspecto fundamental para explicar o maior acometimento por TB em homens, quando comparados às mulheres, além da baixa procura dos homens pelos serviços de saúde. Dessa forma, os achados da pesquisa ratificam o que foi encontrado em outros estudos, assim como o padrão nacional da apresentação da $\mathrm{TB}^{8-12}$.

Segundo Hino et al. ${ }^{13}$, em países desenvolvidos, a maior incidência de TB é nas pessoas acima dos 50 anos, o que difere dos países em desenvolvimento. Nestes, a enfermidade é mais incidente em adultos jovens, ou seja, a população economicamente ativa, resultando em comprometimento da parcela produtiva da comunidade.

Tabela 1. Número e percentagem de notificações de TB de pacientes residentes nas áreas adscritas à USF Eduardo Angelim, no município de Belém-PA, no período de 2010 a 2015, de acordo com as variáveis de idade, sexo, raça / cor e escolaridade

\begin{tabular}{|c|c|c|}
\hline Variáveis & Número de notificações & Percentagem de notificações (\%) \\
\hline \multicolumn{3}{|l|}{ Idade do paciente (anos) } \\
\hline $14-25$ & 12 & 27,3 \\
\hline $26-35$ & 09 & 20,5 \\
\hline $36-45$ & 09 & 20,5 \\
\hline $46-55$ & 06 & 13,6 \\
\hline $56-65$ & 06 & 13,6 \\
\hline $66-75$ & 02 & 4,5 \\
\hline \multicolumn{3}{|l|}{ Sexo do paciente } \\
\hline Masculino & 31 & 70,5 \\
\hline Feminino & 13 & 29,5 \\
\hline \multicolumn{3}{|l|}{ Raça/Cor do paciente } \\
\hline Parda & 34 & 77,3 \\
\hline Branca & 07 & 15,9 \\
\hline Preta & 03 & 6,8 \\
\hline \multicolumn{3}{|l|}{ Grau de Escolaridade do paciente } \\
\hline Analfabeto & 02 & 4,5 \\
\hline $1-4^{a}$ série incompleta do EF & 07 & 15,9 \\
\hline $4^{\mathrm{a}}$ série completa do EF & 01 & 2,3 \\
\hline 5- $8^{\mathrm{a}}$ série incompleta do EF & 10 & 22,7 \\
\hline EF completo & 04 & 9,1 \\
\hline Ensino Médio incompleto & 06 & 13,6 \\
\hline Ensino Médio completo & 05 & 11,4 \\
\hline Educação superior completa & 01 & 2,3 \\
\hline Ignorado & 08 & 18,2 \\
\hline TOTAL & 44 & 100 \\
\hline
\end{tabular}

Fonte: Secretaria Municipal de Saúde (SESMA), 2016. 


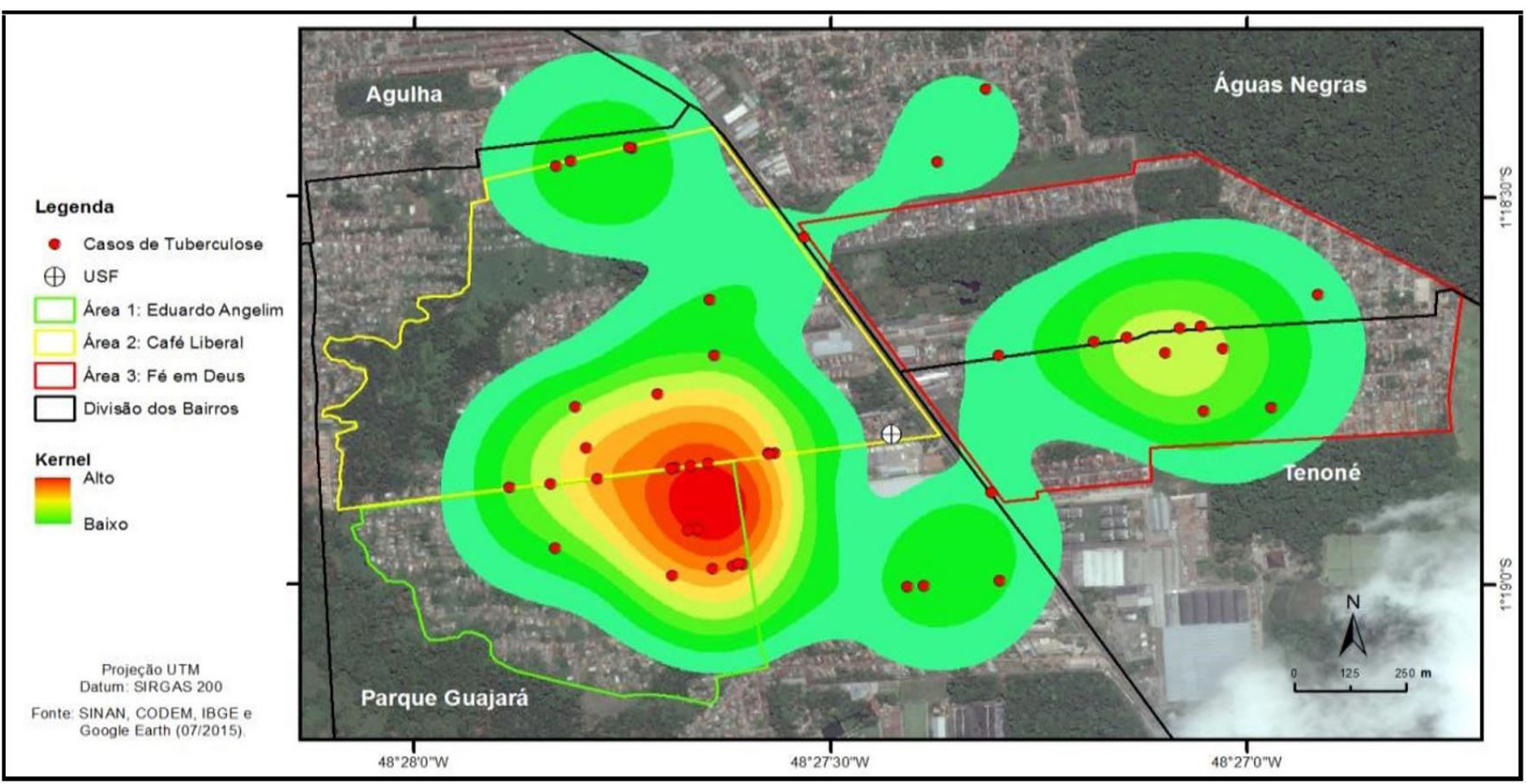

Figura 2. Regiões com maior densidade de notificações de TB nas áreas adscritas à USF Eduardo Angelim, no município de Belém-PA, no período de 2010 a 2015, conforme técnica de Kernel. Fonte: EPIGEO/DSCM/CCBS/UEPA, 2016.

A escolaridade da população afeta o acesso ao conhecimento e a capacidade de compreensão das pessoas, interferindo diretamente na educação e no entendimento acerca da promoção e prevenção das doenças e da terapêutica prescrita. Assim, os doentes de TB, por apresentarem, predominantemente, baixa escolaridade, mostram-se em condições desfavoráveis e, por conta disso, necessitam de mais investimentos do governo direcionados a eles ${ }^{8-10}$.

Um dos principais achados deste estudo foi a intensa heterogeneidade com que a TB se distribuiu espacialmente. A maior incidência foi localizada na área Eduardo Angelim. Isso se enquadra na grande relação entre a TB, o espaço e sua organização, com achados de outros estudos ${ }^{11-13}$. A Figura 2, baseada na técnica de Kernel, permitiu identificar onde os eventos estavam concentrados, evidenciando desigualdades na área analisada e a aglomeração espacial dos $\operatorname{casos}^{12}$.

Neste estudo, observou-se maior ocorrência e densidade de casos de TB nas áreas adscritas à USF que apresentam carência socioeconômica. As desigualdades de habitação, distribuição de renda e acesso à educação afetam o adoecimento da população por resultarem na desvantagem de alguns grupos quanto à oportunidade de serem e de se manterem sadios ${ }^{9-11}$.

Em relação ao mapeamento geográfico dos casos, uma das limitações encontradas foi a incompletude dos dados informados na USF e na SESMA, principalmente nos registros de endereços. Isso é problemático, dado que estas informações são fundamentais para estimular a estratégia e a criação de políticas públicas, na medida em que ajuda na apreensão da realidade, monitoramento de doenças e agravos distribuídos no território ${ }^{8}$. Nesse contexto é imprescindível sensibilizar gestores e profissionais de saúde para a relevância da informação em saúde, pois ela gera subsídios para a tomada de decisão, possibilitando melhorias para a comunidade e evitando a disseminação da tuberculose ${ }^{13}$.

\section{Conclusão}

Este estudo permitiu conhecer a distribuição espacial dos casos novos de TB nas áreas adscritas à USF em estudo, e reconhecer que a doença não ocorre com a mesma intensidade em todos os grupos populacionais. O mapeamento geográfico torna-se importante para o reconhecimento das áreas de vulnerabilidade para direcionar políticas de enfrentamento e buscar contribuir para o controle da doença.

Espera-se, assim, contribuir para o fortalecimento das ações de combate à TB, direcionar os gestores no planejamento, no monitoramento e na avaliação das ações de saúde. Além disso, permite direcionar políticas 
de enfrentamento e de controle da doença, como a busca ativa dos doentes e comunicantes, notificação dos casos, educação em saúde, acompanhamento e supervisão do tratamento.

\section{Agradecimentos}

Ao Laboratório de Epidemiologia e Geoprocessamento da Amazônia (EpiGeo) da Universidade do Estado do Pará pelo apoio durante a interpretação dos dados e realização do mapeamento geográfico. À Secretaria Municipal de Saúde (SESMA) de Belém-PA pela agilidade e apoio durante a coleta de dados.

\section{Referências}

1. Organização das Nações Unidas no Brasil. Tuberculose causa 5 mil mortes por dia no mundo, alerta OMS [Internet]. Brasil: Nações Unidas no Brasil; 2017 [citado em 2018 Jun 7]. Disponível em: https://nacoesunidas.org/tuberculose-causa-5-mil-mortes-por-dia-no-mundo-alerta-oms/

2. Peruhype RC, Acosta LMW, Ruffino-Netto A, Oliveira MMC, Palha PF. Distribuição da tuberculose em Porto Alegre: análise da magnitude e co-infecção tuberculose-HIV. Rev Esc Enferm USP [Internet]. 2014 Ago-Dez [citado em 2016 Mai 6];48(6):1035-43. Disponível em: http:// www.scielo.br/pdf/reeusp/v48n6/pt_0080-6234-reeusp-48-06-1035.pdf.

3. Brunello MEF, Chiaravalloti No F, Arcêncio RA, Andrade RLP, Magnabosco GT, Villa TCS. Áreas de vulnerabilidade para co-infecção HIVaids/TB em Ribeirão Preto, SP. Rev Saúde Públ [Internet]. 2011 Abr [citado em 2016 Mai 8];45(3):556-63. Disponível em: http://www.scielo. $\mathrm{br} / \mathrm{pdf} / \mathrm{rsp} / \mathrm{v} 45 \mathrm{n} 3 / 2331 . \mathrm{pdf}$.

4. World Health Organization. Global Tuberculosis Report 2014 [Internet]. Geneva: World Health Organization; 2014 [citado em 2016 Abr 20]. 4 p. Disponível em: http://www.who.int/tb/publications/global_report/archive/en/.

5. Brasil. Ministério da Saúde. Secretaria de Vigilância em Saúde. Boletim Epidemiológico. 2015;45(9):1-5.

6. Brasil. Ministério da Saúde. Secretaria de Atenção à Saúde. Departamento de Atenção Básica. Tuberculose na atenção primária à saúde. 2. ed. Brasília: Ministério da Saúde; 2011. 131 p. (Série A. Normas e Manuais Técnicos).

7. Brasil. Ministério da Saúde. Secretaria de Vigilância em Saúde. Departamento de Vigilância Epidemiológica. Manual de recomendações para o controle da tuberculose no Brasil. Brasília: Ministério da Saúde; 2011.

8. Hino P, Villa TCS, Cunha TN, Santos CB. Distribuição espacial de doenças endêmicas no município de Ribeirão Preto (SP). Ciênc Saúde Colet [Internet]. 2011 Mar-Abr [citado em 2016 Abr 20];16(Supl 1):1289-94. Disponível em: http://www.scielo.br/pdf/csc/v16s1/a62v16s1.pdf.

9. Brasil. Ministério da Saúde. Secretaria de Vigilância em Saúde. Boletim Epidemiológico. 2016;47(13):1-15.

10. Araujo KMFA, Figueiredo TMRM, Gomes LCF, Pinto ML, Silva TC, Bertolozzi MR. Evolução da distribuição espacial dos casos novos de tuberculose no município de Patos (PB), 2001-2010. Cad Saúde Colet [Internet]. 2013 Ago-Set [citado 2016 Abr 20];21(3):296-302. Disponível em: http://www.scielo.br/pdf/cadsc/v21n3/v21n3a10.pdf.

11. Barbosa IR, Costa ICC. Aspectos epidemiológicos da tuberculose no município de Natal. Rev Enferm UFPI [Internet]. 2013 Jun-Set [citado em 2016 Abr 20];2(2):14-20. Disponível em: http://www.ojs.ufpi.br/index.php/reufpi/article/viewFile/987/pdf.

12. Almeida MG, Barbosa DRM, Almeida DFS. Epidemiologia e distribuição espacial de casos notificados de tuberculose multirresistente (TBMR) no Brasil, 2008-2012. Rev Epidemiol Control Infect [Internet]. 2013 Out [citado em 2017 Jun 17];3(4):117-22. Disponível em: https://online. unisc.br/seer/index.php/epidemiologia/article/viewFile/3564/3251.

13. Hino P, Takahashi RF, Bertolozzi MR, Egry EY. A ocorrência da tuberculose em um distrito administrativo do município de São Paulo. Esc Anna Nery (impr.). 2013 Jan-Mar;17(1):153-9.

\section{Autor correspondente}

Bruno de Oliveira Santos

Conjunto Império Amazônico, Passagem Getúlio Vargas, Souza

CEP 66613-080, Belém, PA, Brasil

Tel: (91) 98127-0532

E-mail: broliveira17@bol.com.br

Informação sobre os autores

BOS, TVRB, LAL e MPR são acadêmicos do curso de medicina pela Universidade do Estado do Pará.

RJPSG é doutor em doutor em Biomedicina.

CRM é enfermeira; mestranda no Programa de Pós-graduação em Epidemiologia e Vigilância em Saúde pelo Instituto Evandro Chagas.

\section{Contribuição dos autores}

BOS, TVRB e CRM contribuíram na elaboração do estudo, na coleta e análise de dados e na escrita final do artigo; RJPSG, LAL e MPR contribuíram para análise de dados e revisão final do artigo. 